\title{
OPEN-END YARN PROPERTIES PREDICTION USING HVI FIBRE PROPERTIES AND PROCESS
} PARAMETERS

\author{
Hanen Ghanmi ${ }^{1,2}$, Adel Ghith ${ }^{2,3}$, Tarek Benameur ${ }^{1}$ \\ 1 University of Monastir, National Engineering School, Laboratory of Mechanic Engineering LGM_MA05, 5019 Monastir, Tunisia \\ ${ }^{2}$ University of Monastir, National Engineering School, Department of Textile Engineering, ENIM, 5019 Monastir, Tunisia \\ ${ }^{3}$ University of Monastir, National Engineering School, Research Unit Automatic Image and Signal Processing, 5019 Monastir, Tunisia \\ E-mail: ghanmihan@voila.fr
}

\begin{abstract}
:
This article provides three models to predict rotor spun yarn characteristics which are breaking strength, breaking elongation and unevenness. These models used noncorrelated raw material characteristics and some processing parameters. For this purpose, five different cotton blends were processed into rotor spun yarns having different metric numbers (Nm10, Nm15, Nm18, Nm22, Nm30 and Nm37). Each count was spun at different twist levels. Response surface method was used to estimate yarn quality characteristics and to study variable effects on these characteristics. In this study, predicting models are given by the analysis of response surface after many iterations in which nonsignificant terms are excluded for more accuracy and precision. Itwasshownthatyarncount, twistand sliverpropertieshadconsiderableeffectsontheopen-endrotorspunyarnproperties. This study can help industrial application since it allows a quality management-prediction based on input variables such as fibre characteristics and process parameters.
\end{abstract}

\section{Keywords:}

HVI characteristics, open-end rotor yarn, process parameters, response surface method

\section{Introduction}

Rotor spinning, which was invented in 1965, has a high percentage of production around the world [8]. Nowadays, this spinning process still has a large production in the world, because, compared with ring spun yarn, rotor spun yarn has the advantage of good evenness, less count variation and imperfections. Furthermore, rotor spinning, compared with ring spinning method, exhibits less energy cost because of less machinery involved in the yarn production process. Also, yarn breakage rate in rotor spinning is lower. This fact enhances production and yarn quality.

The study of rotor yarns has attracted the interest of many researchers in different sides, such as yarn structure [5,6], rotor spun yarn tensile properties $[1,4,7,10]$, the effect of twist level and other process parameters on yarn properties as well as the prediction of the mechanical behaviour [9].

Generally, yarn quality is defined in two main characteristics which are tenacity and elongation. Indeed, breaking strength is an important quality characteristic that affects the determination of its application possibilities. Nevertheless, customers usually require much more quality criteria like yarn unevenness expressed by mass variation coefficient. This parameter is defined as the irregularity of the yarn linear density in the direction of its length. These properties are influenced by raw fibre characteristics, process variables and machine parameters.
Statistical models were the first approaches used in the textile engineering field to explore the relationship between variables and yarn properties and optimise them [3]. However, regression analysis has the drawback that it is limited by the problem of nonlinearity. But, we can avoid this disadvantage using response surface method. This methodology is a mathematical technique that has been successfully used to develop processes [2]. It was also used to model processes and to optimise processing parameters and rotor spun yarn quality.

Thus, the purpose of this study is to develop response surface regression models for the prediction of cotton open-end rotor spun yarn strength, elongation and unevenness using fibre properties, slivers characteristics, yarn count and yarn twist.

The novelty of our research is that the conceived models are more practical and more powerful since they contain less input number than other models seen in different researches and all nonsignificant terms are removed as it will be indicated in the next section.

\section{Materials and methods}

\section{Sample yarn production and experimental}

The data used in the predicting models were collected from cotton yarns produced on the same rotor machine using five different cotton blends taken from various origins. Two second 
draw slivers were furnished for each blend with metric number of 0.206 and 0.222 .

The correlation coefficients between the fibre properties used in the study were determined before the regression analysis. The results revealed that there is a strong correlation between some factors. Therefore, we will investigate only two fibre properties which are SFI and Str.

Rotor spun yarns having metric number 15 are given by the first sliver, other yarns having metric number are given by the second sliver. Furthermore, each count was spun at different twist levels as given in Table 1.

Table 1. Process characteristics of produced yarns

\begin{tabular}{|c|c|}
\hline Metric numbers & Twist levels \\
\hline 10 & 400 \\
\hline $15,18,20$ & 400 \\
\hline $15,18,20$ & 500 \\
\hline $15,18,20$ & 600 \\
\hline $15,18,20$ & 700 \\
\hline 30 & 600 \\
\hline 30 & 700 \\
\hline 30 & 1000 \\
\hline 37 & 600 \\
\hline 37 & 700 \\
\hline 37 & 1000 \\
\hline
\end{tabular}

Yarn cones were selected randomly from the lots of each yarn and tested for their characteristics after conditioning in a standard atmosphere for 24 hours. Yarn tensile properties were measured by Uster Tensorapid 3 which provides strength, elongation, RKM and work. Similarly, Uster tester 3 was used to determine $\mathrm{CVm}$, total imperfections (thin places, thick places and neps) and hairiness. Summary statistics of fibres, slivers and yarns factors are given in the following table.

\section{Model construction and analyses using response surface method}

After creating response surface design, we have to analyse it in order to build the predicting model which contains three types of terms: linear terms, squared terms and interaction terms. As a consequence, the general relationship between the response $y$ and the different parameters $\left(x_{i}\right.$ and $\left.y_{i}\right)$ will be expressed as follows:

$$
y=a_{0}+\sum_{i=1}^{n} a_{i} x_{i}+\sum_{i=1}^{n} a_{i i} x_{i}^{2}+\sum_{i=1}^{n-1} \sum_{j=2}^{n} a_{i j} x_{i j}
$$

where $a_{0}=$ the constant term, $a_{i}=$ the coefficients of main factor effects, $a_{i i}=$ the coefficient of quadratic effect, $a_{i j}=$ the coefficient of interaction effects and $n=$ the number of factors chosen (six in this case).

However, to get a more appropriate predicting model, we adopted several iterations in which we have to exclude some insignificant terms having $p$-values more than 0.05 and we must be careful not to decrease adjusted $R^{2}$. This process is given in detail in figure 1. The statistical analyses were performed using the minitab 16 software program.

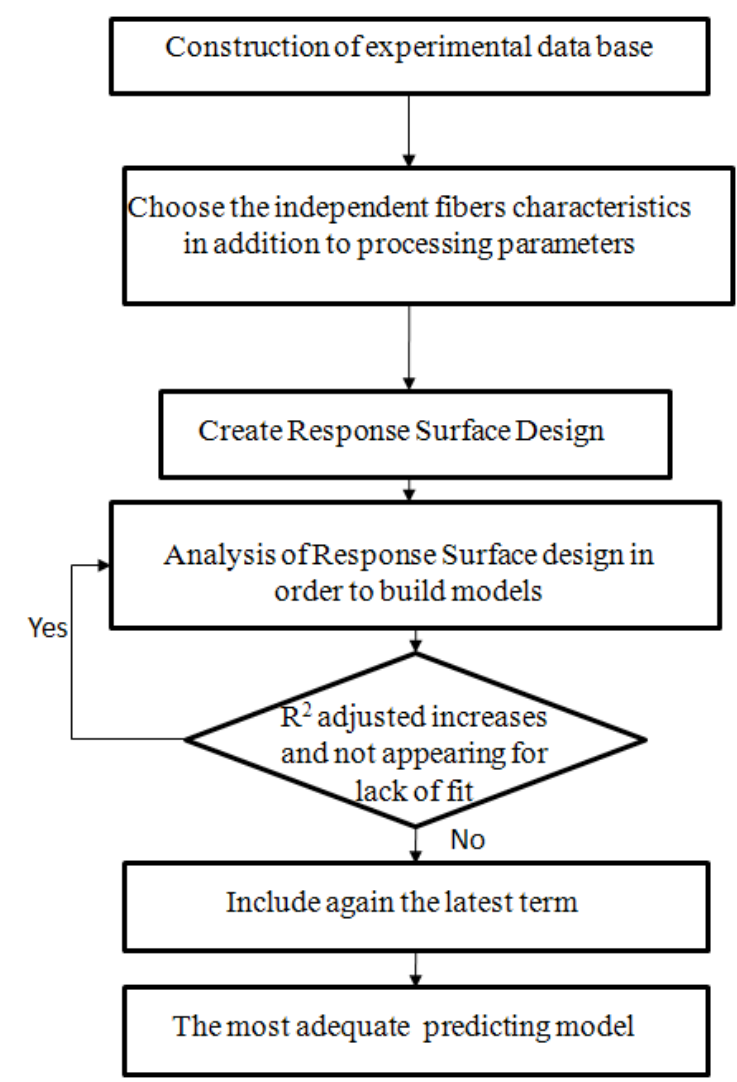

Figure 1.Research flowchart

Table 2. Fibres, slivers and yarns properties statistics summary

\begin{tabular}{|c|c|c|c|c|}
\hline Factor & Symbol & Minimum-value & Mean-value & Maximum-value \\
\hline Short fibre Index & SFI & 7.9 & 9.12 & 10.7 \\
\hline Fibers strength & Str & 25.5 & 28.100 & 31.2 \\
\hline Yarn metric number & Nm & 10 & 37 & 22.04 \\
\hline Twist level & Twist & 400 & 1000 & 598.461 \\
\hline Sliver metric number & NmR & 0.206 & 0.222 & 0.218 \\
\hline Sliver unevenness & RgR & 2.52 & 2.94 & 2.6533 \\
\hline
\end{tabular}




\section{Result and discussion}

\section{Study of yarn strength}

The response surface regression equation predicting the yarn strength is given as follows:

Strength $(K g f)=-1.7538-0.1299 S F I+0.0821 S t r$

$-0.1960 N m+0.0039 T$ wist $+12.8298 N m R+0.0022 \mathrm{Nm}^{2}$

$+0.0034 S F I \times N m-0.0001 S t r \times$ Twist

This model has a very high predictive power since we have $\mathrm{R}^{2}=$ 97.8 as given by figure 2 :

\section{Srength Regression Coefficient}

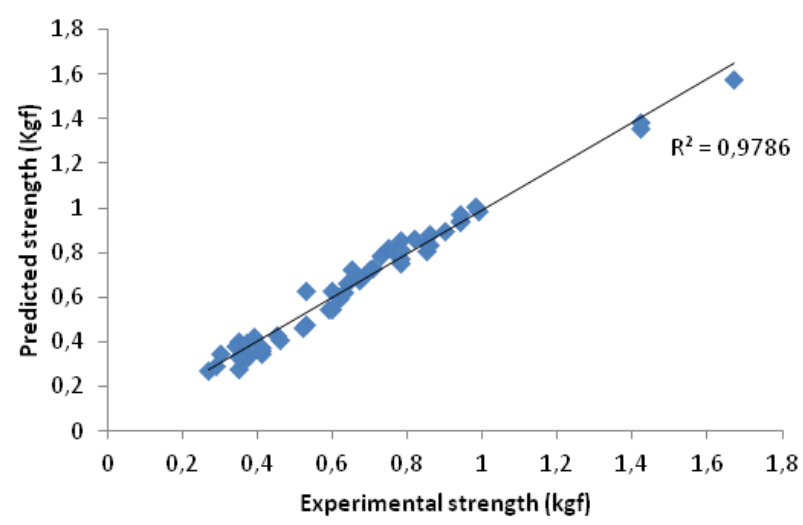

Figure 2. Experimental and predicted values of breaking elongation

As depicted in table 3, the most important parameter affecting yarn strength is the metric number $(T=-14.366)$ and it has a negative effect. Thus, when we have a coarser yarn, we will have more fibres in cross section and so more fibres will participate in yarn strength. Furthermore, the effect of metric number depends on short fibre index and twist. Then, we have the sliver count $(T=10.554)$, so, thicker sliver gives stronger yarns. Moreover, SFI and Str have both significant effects on yarn strength but these two effects are opposite. Indeed, stronger fibres give stronger yarns. But, the shorter fibres are, the weaker the yarn becomes.

Besides, the twist level has, also, a positive and a significant effect on breaking strength. Thus, with a higher twist level, fibre friction increases and so more fibres would participate in yarn strength. The effects of these several factors on yarn strength are shown in surface plots figure.

It is evident from these plots that twist level and metric number have both quadratic effects on yarn strength. These results agree with T-values for Twist ${ }^{2}$ and $\mathrm{Nm}^{2}$ given by Table 3. Also, Figure $3 \mathrm{~b}$ shows that yarn strength rises until about $800 \mathrm{turns} / \mathrm{m}$ then it decreases.

\section{Study of yarn elongation}

A more appropriate response surface model predicting yarn elongation is given by the following equation:

$$
\begin{aligned}
& \text { Elongation }(\%)=-6.3400-0.5289 S F I \\
& +0.0609 S t r-0.2519 \mathrm{Nm}+3.1659 \mathrm{RgR} \\
& +0.0040 \text { Twist }+49.2076 \mathrm{NmR}+0.0023 \mathrm{Nm}^{2}
\end{aligned}
$$

According to table 4, breaking elongation is highly influenced by twist level. So, elongation increases with higher twist and decreases with lower one. In addition, metric number has an important and a negative effect on breaking elongation. So, coarser yarns have more elongation than finer yarns. The metric number of roving has also a considerable effect $(T=3.402)$.

As far as fibre characteristics are concerned, short fibre index has a negative effect on yarn elongation ( $T=-0.867$ ). However, fibre strength affects positively breaking elongation.

Furthermore, yarn count has a quadratic effect (T-value for $\mathrm{Nm}^{2}=2.227$ ) indicating that the relationship between this parameter and breaking elongation follows a curved line.

Table 3. Regression coefficients, $T$ and $P$ values of model for yarn strength

\begin{tabular}{|c|c|c|c|}
\hline Terms & Coefficient & T & P \\
\hline Constant & -1.7538 & -2.466 & 0.018 \\
\hline SFI & -0.1299 & -4.553 & 0.00 \\
\hline Str & 0.0821 & 4.136 & 0.00 \\
\hline Nm & -0.1960 & -14.366 & 0.00 \\
\hline Twist & 0.0039 & 4.004 & 0.00 \\
\hline NmR & 12.8298 & 10.554 & 0.00 \\
\hline Nm & 0.0022 & 12.968 & 0.00 \\
\hline Twist ${ }^{2}$ & 0.0000 & -3.415 & 0.001 \\
\hline SFI*Nm & 0.0034 & 2.853 & 0.007 \\
\hline Str*Twist & -0.0001 & -2.240 & 0.030 \\
\hline NmTwist & 0.0000 & 1.526 & 0.135 \\
\hline
\end{tabular}


The following figure shows the scatter plot of the predicted and experimental values of yarn elongation and the coefficient $R^{2}$

\section{Study of CVm}

Response surface model estimated for yarn unevenness is given by the following equation:

$$
\begin{aligned}
& C V_{m}=-5.8596+0.1686 S F I+0.4246 S t r \\
& +0.8323 N m+13.9518 N m R+0.0023 N^{2} \\
& -0.0280 S t r \times N m
\end{aligned}
$$

The above table shows that, as expected, yarn count has the most significant effect $(T=4.640)$. Therefore, coarser yarns have the best evenness. Moreover, the relationship between yarn unevenness and $\mathrm{Nm}$ follows a curved line and depends on fibre strength characteristic as given by figure 5 .

Among fibre properties, strength has a significant and a positive impact on $\mathrm{CVm}(\mathrm{T}=3.125)$. Also, short fibre index has a positive effect on $\mathrm{CVm}$. So, the longer fibres are, the better evenness is.

Figure 6 shows the scatter plot of the predicted and experimental values of yarn unevenness and the coefficient $R^{2}$

\section{Elongation Regression coefficient}

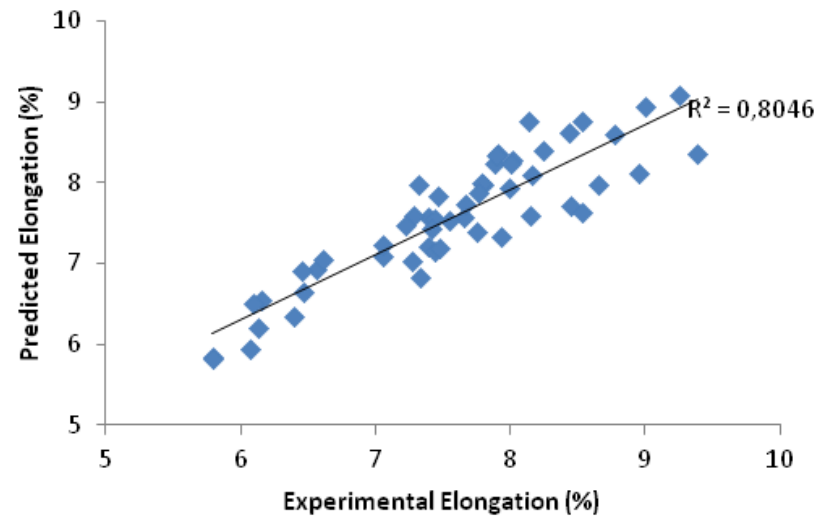

Figure 4. Experimental and predicted values of breaking elongation a)

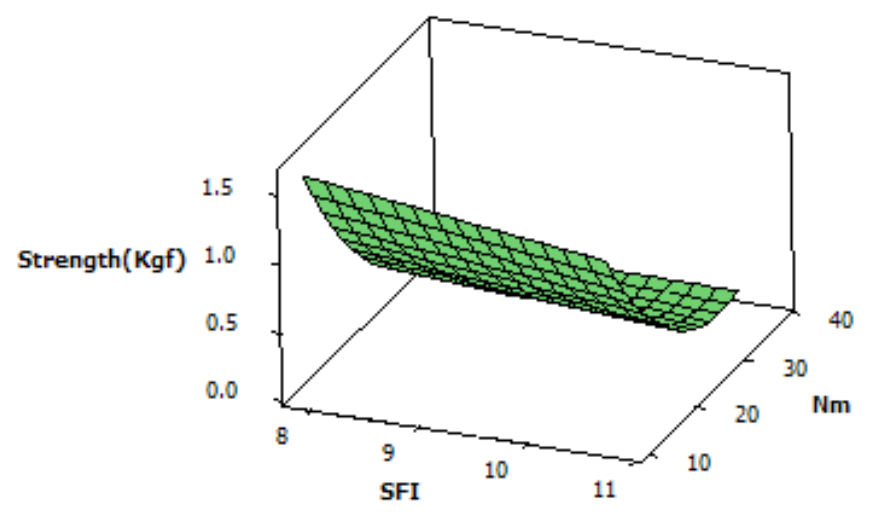

b)

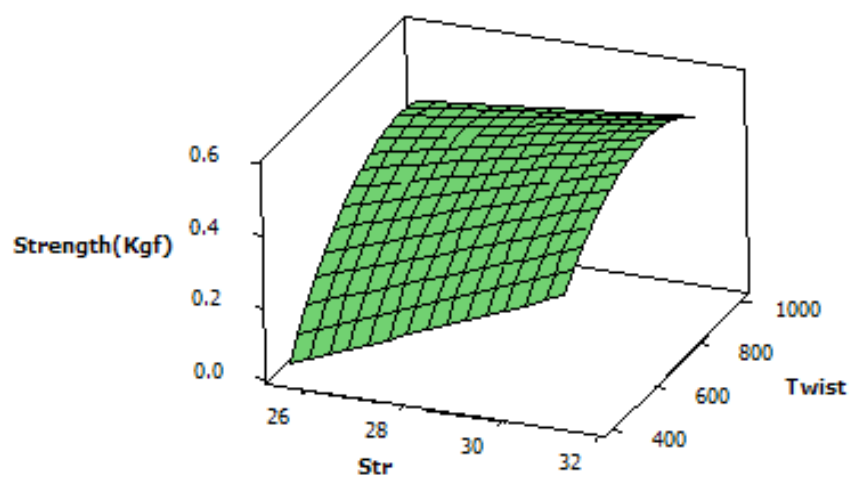

c)

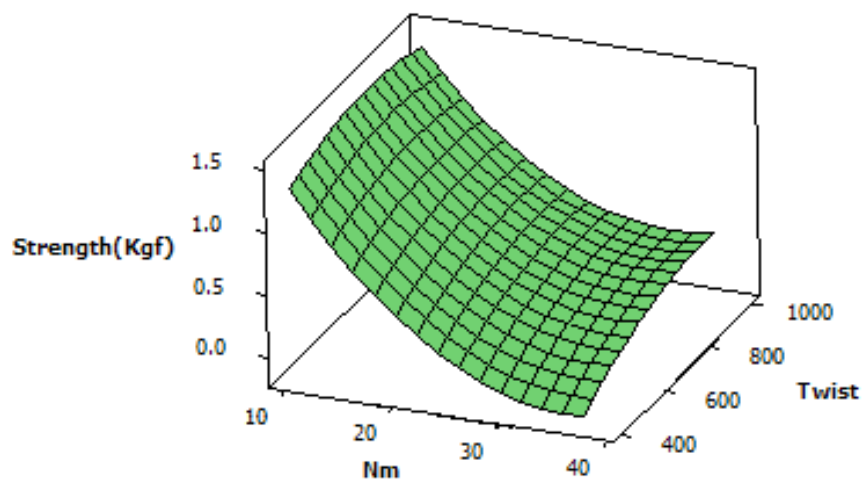

Figure 3. Effects of (a) SFI and Nm (b) Str and twist (c) Nm and twist on yarn strength

Table 4. Regression coefficient, $T$ and $P$ values for yarn elongation

\begin{tabular}{|c|c|c|c|}
\hline Terms & Coefficient & $\mathbf{T}$ & $\mathbf{P}$ \\
\hline Constant & -6.3400 & -1.819 & 0.076 \\
\hline $\mathrm{SFI}$ & -0.5289 & -0.867 & 0.391 \\
\hline $\mathrm{Str}$ & 0.0609 & 1.091 & 0.281 \\
\hline $\mathrm{Nm}$ & -0.2519 & -4.721 & 0.000 \\
\hline $\mathrm{RgR}$ & 3.1659 & 1.245 & 0.220 \\
\hline Twist & 0.0040 & 8.868 & 0.000 \\
\hline $\mathrm{NmR}$ & 49.2076 & 3.402 & 0.001 \\
\hline $\mathrm{Nm}^{2}$ & 0.0023 & 2.227 & 0.031 \\
\hline
\end{tabular}


Table 5. Regression coefficients, $T$ and $p$ values for yarn $\mathrm{CVm}$

\begin{tabular}{|c|c|c|c|}
\hline Terms & Coefficient & $\mathbf{T}$ & $\mathbf{P}$ \\
\hline Constant & -5.8596 & -1.277 & 0.208 \\
\hline $\mathrm{SFI}$ & 0.1686 & 1.960 & 0.056 \\
\hline $\mathrm{Str}$ & 0.4246 & 3.125 & 0.003 \\
\hline $\mathrm{Nm}$ & 0.8323 & 4.640 & 0.000 \\
\hline $\mathrm{NmR}$ & 13.9518 & 1.363 & 0.180 \\
\hline $\mathrm{Nm}^{2}$ & 0.0023 & 2.237 & 0.030 \\
\hline $\mathrm{Str}^{*} \mathrm{Nm}$ & -0.0280 & -4.810 & 0.000 \\
\hline
\end{tabular}

\section{Conclusion}

Open-end rotor spun yarn characteristics are influenced by $\mathrm{HVI}$ fibre characteristics and process parameters which are sliver properties, metric number and twist level. In this study, we tried to predict three important yarn characteristics which are strength, elongation and $\mathrm{CVm}$ with response surface regression method using these parameters. The predicting models have good prediction performance as the regression coefficient $R^{2}$-values of these models are high and have a greater significance.

Our study shows that yarn count and twist level are the most decisive factors for yarn properties. Also, sliver number and regularity as well as the two selected fibre properties all have considerable effects.

This paper shows that the statistical approach gives good results and takes into consideration the nonlinearity between predictors. So, this method is still important and very useful as it indicates explicitly the relationship between responses and variables unlike the artificial neural network approach.

In addition, this study will be extended to find the optimal processing parameters and fibre characteristics for the openend yarn properties optimisation and look for a new yarn quality expression that investigates the three properties studied in this article.

\section{References}

[1] Arain F A, Tanwari A, Hussain T \& Z A Malik, (2012). Multiple Response Optimization of Rotor Yarn for Strength, Unevenness, Hairiness and Imperfections. Polymers and Fibers, vol13, $N^{\circ} 1,118-122$.

[2]Atkinson A.C. and Donev A.N., (1992). Optimum Experimental Designs, Oxford university press, Oxford, 132-189.

[3] Erol R \& Sagbas A, (2009). Multiple Response Optimization of Staple-Yarn Production Process of Hairiness, Strength and Cost. Fibers and Textiles in Eastern Europe, 17, 4042.

[4] Kuo C F J , Wei H J, Huang C C \& Su T L, (2010). Processing Parameters Optimization of Multiple Quality Characteristics of Open-End Rotor Spinning Process for Bamboo Charcoal and CVC Blended Fibers. Fibers and Polymers, vol11, N6, 891-898.

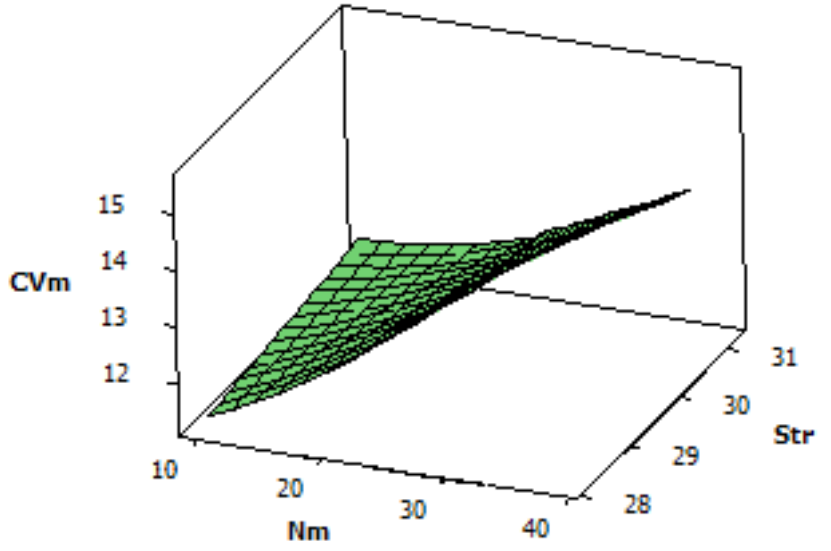

Figure 5. Effect of $\mathrm{Nm}$ and fibre strength on $\mathrm{CVm}$

\section{Regression coefficient for CVm}

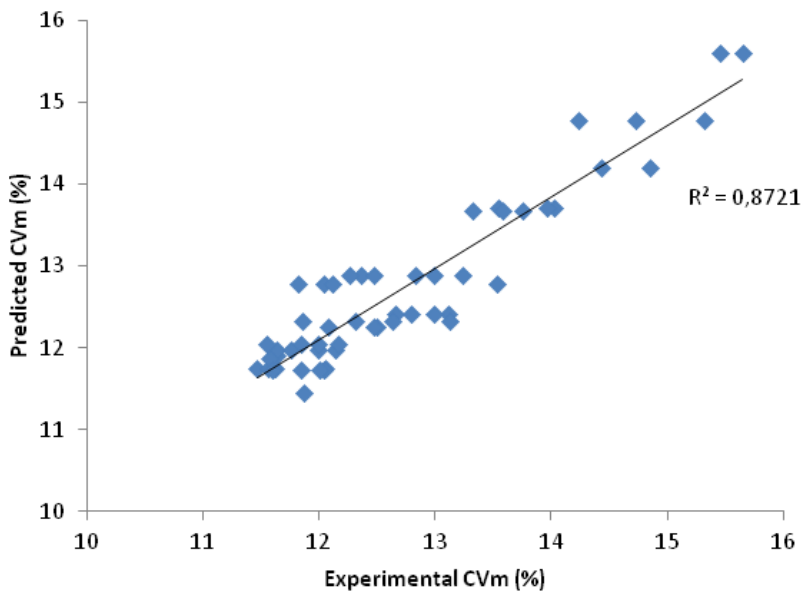

Figure 6. Experimental and predicted values of yarn $\mathrm{CVm}$

[5] Lawrence C A \& Finikopulos E, (1992).Factors Affecting changes in The Structure and Properties of Open-End Rotor Yarns. Indian Journal of Fiber \& Textile Research, vol17 (4), 201-208.

[6] Lord P R \& Grady P L, (1976). The Twist Structure of OpenEnd yarns. Textile Research Journal, 46, 123-129.

[7] Moghassem A, Fallahpour A \& Shanbeh M, (2012).An Intelligent Model to Predict Breaking Strength of Rotor Spun Yarns Using Gene Expression Programming. Journal of Engineered Fibers and Fabrics, vol7, Issue2, 1-10. 
[8] Padamanabhan A R, (1989). A Comparative Study of the Properties of Cotton Yarns Spun on the DREF-3 and Ring and Rotor Spinning Systems. Journal of textile Institute, 80, 555-562.

[9] Sami B A \& Naima H, (2009).Prediction of the Behavior of Open-End and Ring Spun yarns. Journal of Applied Sciences 9(8), 1466-1473.
[10] Sharma I C, Gupta N K, Agarwal B R \& Pataik N R, (1987). Effect of twist factor and stitch length of open-end spun cotton yarn on properties of Rib Knitted fabrics. Textile research journal, 57, 73-81. 